\title{
Curriculare Mehrsprachigkeit am Beispiel des Deutschen in Estland
}

MERJE MILISTE

\begin{abstract}
Curricular Multilingualism using the Example of German in Estonia. In Estonia, as everywhere in Europe, multilingualism and plurilingual competence are widely discussed topics. The present article looks at curricular multilingualism in Estonia and highlights the effect of political changes on language curricula at school and on learning multiple languages. The article shows how German as the first foreign language can contribute to plurilingualism.
\end{abstract}

Keywords: plurilingualism; curriculum; foreign language learning; German as a foreign language

\section{Curriculare Mehrsprachigkeit}

Der deutsche Begriff „Mehrsprachigkeit” wird als unscharf angesehen, weil darunter unterschiedliche Perspektiven auf die sprachliche Kompetenz, d.h. sowohl Multilingualität als auch Plurilingualität zusammengefasst werden (Neuner 2004: 173). Deshalb findet man in der einschlägigen Literatur zahlreiche Versuche, den vagen Begriff der Mehrsprachigkeit durch präzisierende Attribute zu spezifizieren und zu differenzieren. So unterscheidet zum Beispiel Königs (2003) je nach Lernort und Kommunikationssituation 18 Typen von Mehrsprachigkeit.

Den Begriff der curricularen Mehrsprachigkeit, der im Fokus dieses Beitrags steht, haben Hans-Jürgen Krumm und Gerhard Neuner geprägt. Darunter wird das Angebot unterschiedlicher Optionen im Curriculum für die Entfaltung individueller Mehrsprachigkeitsprofile verstanden (Neuner 2004). Krumm (2003: 45) geht noch einen Schritt weiter und fordert eine Vielfalt im sprachlichen Curriculum, wo Kurse sich ergänzen und aufeinander aufbauen. Inwiefern und wie mehrsprachendidaktische Ansätze in der Unterrichtspraxis umgesetzt werden, soll jedoch an einer anderen Stelle diskutiert werden. Im Folgenden wird am Beispiel Estlands nachgezeichnet, wie sich das Politische auf die Mehrsprachigkeit in den nationalen Lehrplänen ausgewirkt hat. 
2 Wendepunkte der curricularen Mehrsprachigkeit im estnischen Bildungswesen

Da die Esten mit einer Sprecherzahl von einer knappen Million ein von der Bevölkerungszahl her eher kleines Volk sind, wird die Handlungsfähigkeit der Individuen in mehreren Sprachen hierzulande auf bildungspolitischer Ebene sehr geschätzt (Haridus- ja Teadusministeerium 2020: 3). Schon am Anfang des 20. Jahrhunderts war der Unterricht in zwei, drei oder vier Fremdsprachen Realität (Põldvere 2003: 28). Welche Sprachen in der Gesellschaft und somit auch im Bildungskontext Konjunktur haben, hängt jedoch vor allem von politischen und sozialen Machtverhältnissen ab.

Das estnische Volk blickt auf eine Geschichte zurück, die über Jahrhunderte hinweg von fremden politischen Strukturen und von Fremdkulturen geprägt worden ist (Laar 2017). Seit dem 13. Jahrhundert hat die deutsche Sprache in diesem Gebiet eine wichtige Rolle gespielt: Sie war die Sprache der machthabenden Oberschicht, während die Bauern Estnisch oder Livisch sprachen. Im 18. Jahrhundert galt Deutsch als die grundlegende Schrift- und Behördensprache. Sie war die Unterrichtssprache der höheren Lehranstalten und der Kaiserlichen Universität zu Dorpat. Bis zur zweiten Hälfte des 19. Jahrhunderts war Deutsch auch die Hauptsprache der Presse. In den 1880er Jahren setzten in den baltischen Provinzen Russifizierungsprozesse ein, um die Macht der lokalen deutschen Elite zu reduzieren. Die russische Sprache ersetzte im Jahre 1887 in Estland das Deutsche als Unterrichtssprache in den höheren Lehranstalten und an der Universität. So spricht man noch in der ersten Hälfte des 20. Jahrhunderts von drei lokalen Sprachen in diesem Gebiet: Estnisch, Deutsch und Russisch (Bender 2017).

Vor der ersten Unabhängikeitserklärung der Esten im Jahre 1918 setzte der Unterricht von fremden Sprachen in den allgemeinbildenden Schulen in der 4. Klasse mit 4 Wochenstunden ein. Zu jener Zeit war noch Russisch die erste Fremdsprache, gefolgt von Englisch oder Deutsch als zweite, Deutsch oder Französisch als dritte und Latein als vierte Fremdsprache (Põldvere 2003: 28). Im Jahre 1921 wird der Beginn des Fremdsprachenunterrichts in der ersten unabhängigen Republik Estlands auf die 3. Klasse vorverlegt, wobei nun lediglich Deutsch und Englisch als erste Fremdsprachen zugelassen werden (Põldvere 2003: 28).

Der Nichtangriffsvertrag zwischen Deutschland und der Union der Sozialistischen Sowjetrepubliken im Jahre 1939 hat fatale Folgen auf die staatliche Souveränität aller baltischen Staaten. Der Roten Armee werden Stationierungsrechte im Baltikum eingeräumt und etwa 20000 Deutsch-Balten werden von 1939-1941 unter dem Motto „Heim ins Reich“ aus dem estnischen 
Gebiet umgesiedelt. Die Umsiedlung der Deutsch-Balten schwächt die Position des Deutschen allmählich ab und der Anteil der Deutschlernenden fällt auf fast ein Viertel ab (Mohr 2001: 1683).

Nach dem Zweiten Weltkrieg gehörte Estland der UdSSR an, deren offizielle Verkehrssprache Russisch war, das auf dem gesamten Gebiet des ehemaligen Ostblocks den Status einer lingua franca genoss. Die hervorgehobene Stellung des Russischen im Sprachenkanon der estnischen Lehrpläne war von nun an wegen des politischen Drucks festgeschrieben. Der Russischunterricht setzte in den allgemeinbildenden Schulen mit Estnisch als Unterrichtssprache im zweiten Schuljahr verpflichtend ein und währte dann elf Jahre bis zum Abitur. In der Estnischen Sowjetischen Sowjetrepublik waren zwei Fremdsprachen verpflichtend vorgesehen: Mit dem Erlernen der zweiten Fremdsprache als Pflichtfach (meistens Englisch oder Deutsch) begann man in der Nachkriegszeit in der 5. Klasse mit 4 Wochenstunden, seit 1981 in der 4. Klasse mit 3 Wochenstunden (Põldvere 2003: 30f.). Im Allgemeinen verringerte sich das Gesamtvolumen des Fremdsprachenunterrichts hinter dem Eisernen Vorhang summa summarum von 945 Wochenstunden (im Jahre 1944) in den 1970er Jahren auf 630 und am Ende der 80er Jahre auf 595 Wochenstunden.

Die zweite Unabhängigkeitserklärung der Estnischen Republik am 20. August 1991 hat mehrere curriculare Reformen in Gang gesetzt. Bereits im Schuljahr 1991/1992 wird der obligatorische elf Jahre andauernde Russischunterricht an den allgemeinbildenden Schulen mit Estnisch als Unterrichtssprache außer Kraft gesetzt. Die Präambel des Curriculums für Fremdsprachen verlautet: „Estnische Kultur und Wirtschaft entwickeln sich im regen Informationsaustausch und in intensiver Kooperation mit der Weltkultur und -wirtschaft. Aus diesem Grund wird der Beherrschung von verschiedenen Fremdsprachen, vor allem aber der Beherrschung von Weltsprachen eine besondere Bedeutung beigemessen (Eesti põhi- ja keskhariduse riiklik opppekava 1996). Das neue staatliche Curriculum, das 1996 in Kraft tritt, lässt die Wahl zur ersten Fremdsprache im dritten Schuljahr zwischen Englisch, Deutsch, Französisch und Russisch zu. Welche Fremdsprachen konkret angeboten werden, liegt in der Entscheidungskompetenz der Schulen. An russischsprachigen Schulen wird das Erlernen des Estnischen als Staatssprache im Primarbereich für alle Schüler verpflichtend eingeführt. Das zweite veränderte staatliche Curriculum für die Hauptschule und das Gymnasium von 2002 sieht die Möglichkeit vor, den Beginn des Fremdsprachenunterrichts vorzuverlegen (Põhikooli ja gümnaasiumi riiklik õppekava 2002). In der Forschung besteht darüber Einigkeit: Je früher ein Lerner mit einer fremden Sprache in Berührung kommt, diese erwirbt oder auch lernt, desto besser. 
Das Erlernen einer zweiten Pflichtfremdsprache ist für die Klassenstufe sechs vorgesehen. Als eine wichtige Erneuerung im Curriculum aus dem Jahr 2011 kann man die zweite Fremdsprache zusätzlich zu den vier oben genannten Sprachen auch unter weiteren Fremdsprachen wählen (Gümnaasiumi riiklik óppekava 2011). Eine dritte Fremdsprache und in vereinzelten Gymnasien auch noch eine weitere Fremdsprache wird im Regelfall in der gymnasialen Oberstufe angeboten (Haridus- ja Teadusministeerium 2009, 13f.). Ob die Schulen eine dritte oder gar vierte Wahlmöglichkeit anbieten, ist freigestellt. Um dem Bedarf an fehlenden Deutsch- und Englischlehrenden nachzukommen, wurden viele ehemalige RussischlehrerInnen an der Universität Tartu und an der Pädagogischen Hochschule Tallinn umgeschult.

An dieser Stelle sei noch darauf aufmerksam gemacht, dass im Curriculum von 2002 festgelegt wurde, dass die Schulabgänger mit Hochschulreife das Sprachniveau B2 des Gemeinsamen europäischen Referenzrahmens für Sprachen in der ersten Fremdsprache und etwa das gleiche Sprachniveau auch in der zweiten Fremdsprache erreichen sollten (Põhikooli ja gümnaasiumi riiklik õppekava 2002).

Schließlich soll hier noch auch auf ein extracurriculares Phänomen der Mehrsprachigkeit hingewiesen werden, das der Zerfall des Eisernen Vorhangs in der europäischen Peripherie mit sich gebracht hat. Nach dem Mauerfall lagen Regionen von Estland im deutschen Sendergebiet. Das breite Angebot an Kinderfernsehen der Privatsender wie RTL, Pro7 und Sat1 wirkte auch auf hiesige Kinder und brachte eine Generation von deutschen Fernsehkindern hervor, die ihre rezeptiven sowie produktiven Fertigkeiten in der deutschen Sprache lediglich durch den Konsum deutscher Fernsehsender erworben haben. Denn Fernsehen und Film „wie kaum einem anderen Medium gelingt, positive Emotionen der Rezipienten hervorzurufen. Cartoons machen Kindern einfach Spaß, sie sind in der Regel lustig, was eine intrinsische Motivation ermöglicht. Emotionale Involviertheit wiederum ist dem Lernen neuer Gegenstände und Zusammenhänge sehr zuträglich und unterstützt darum das Erwerben einer Fremdsprache." (Feldmann 2013: 25)

Die Generation der Fernsehkinder ist ein Beweis dafür, dass es möglich ist, im Kindesalter eine fremde Sprache einzig und allein über den Fernsehkonsum zu erlernen, ohne Anleitung, zusätzliche Übungen und ohne Kontrollinstanz. Das steht auch im Einklang mit Tracy Terrells und Stephen Krashens natural approach, welcher besagt, dass wir Sprachen lediglich durch „comprehensible input" in einer angstfreien Atmosphäre erlernen können (Krashen, Terrell 1983: 59). 
Durch die Digitalisierung des estnischen Fernsehens und mit der Streichung der deutschen Programme verschwand leider der Berührungspunkt zwischen den jungen Zuschauern und der deutschen Sprache. Des Weiteren haben estnische Sender an ihrem eigenen Kinderprogramm in estnischer Sprache Verbesserungen vorgenommen und übertragen vermehrt englischsprachiges Fernsehen. Dies verkündete den Vormarsch des Englischen.

\section{Zur Situation der deutsche Sprache im Kontext anderer Fremdsprachen}

Wie im vorangegangenen Abschnitt bereits dargestellt wurde, steht das Deutsche in Estland in einer langen Traditionslinie und der DaF-Unterricht ist fest institutionell verankert. Vor der zweiten Unabhängigkeitserklärung (Schuljahr 1990/91) war das Fach Russisch mit 56,1\% die meistgelernte Fremdsprache, Englisch folgte mit 33,8\% und Deutsch mit 15,6\% (Kangor 2002). Nachdem das Russische als Sprache der ehemaligen Besatzer eine Zeit lang sogar zu den sogenannten „verdrängten Sprachen“ gehört hatte, hat es sich doch in der Rolle der zweiten Pflichtfremdsprache behaupten können. Laut des estnischen Ministeriums für Bildung und Forschung gab es im Schuljahr 2019/2020 125926 Englisch- und 54960 Russischlernende (haridussilm.ee). Die Russische Föderation ist immerhin unser größter Nachbarstaat. Außerdem weist Estland einen hohen Anteil an russischsprachigen MitbürgerInnen auf. Doch gute Russischkenntnisse sind nicht nur in Dienstleistungsberufen wie z.B. Kundenbetreuer oder Krankenpfleger von Belang. Die Bedeutung der Russischkenntnisse nimmt in Bezug auf den lokalen Arbeitsmarkt derzeit wieder zu, weil der Anteil ausländischer Arbeitskräfte aus den ehemaligen GUS-Staaten eine stetig steigende Tendenz zeigt. Im Vergleich zum Jahr 2017 verdreifachte sich die Zahl solcher Arbeitsmigranten im letzten Jahr und betrug 18000 (Orav 2020).

Es soll jedoch erwähnt werden, dass sich immer mehr Arbeitgeber über mangelnde Russischkenntnisse der jüngeren Generationen beschweren (Haridus- ja Teadusministeerium 2017: 13, Tarvas 2017). Wie der Tabelle 1 zu entnehmen ist, erreichen tatsächlich 10\% der Prüflinge das vom staatlichen Curriculum für Gymnasien festgelegte Leistungsniveau B1, d.h. das Niveau der selbständigen Sprachverwendung, nicht. Auf dem Arbeitsmarkt sind sie den Absolventen russischsprachiger Schulen unterlegen. 
MILISTE

Tabelle 1. Leistungsniveau in staatlichen Abiturprüfungen im Schuljahr 2019 (Innove 2019: 4)

\begin{tabular}{lllllll}
\hline Sprache & Zahl & $<$ B1 & B1 & B2 & C1 & C2 \\
\hline Englisch & 7855 & $14,7 \%$ & $22,1 \%$ & $51 \%$ & $8,7 \%$ & $3,5 \%$ \\
Deutsch & 176 & $22,6 \%$ & $25 \%$ & $27,8 \%$ & $25,6 \%$ & $0 \%$ \\
Russisch & 229 & $10 \%$ & $16,6 \%$ & $73,4 \%$ & $0 \%$ & $0 \%$ \\
Französisch & 142 & $9,1 \%$ & $52,8 \%$ & $37,3 \%$ & $0 \%$ & $0 \%$ \\
\hline
\end{tabular}

An dieser Stelle möchte ich CLIL (Content and Language Integrated Learning - Integriertes Lernen von Inhalt und Sprache) als eine Lösung für das Problem der schwachen Russischkenntnisse vorschlagen. Dieser Bildungsansatz wird seit mehr als 20 Jahren an russischsprachigen Schulen Estlands im Estnischunterricht eingesetzt (Võlli 2018) und könnte sich auch im Russischunterricht an estnischsprachigen Bildungsanstalten bewähren. Eine weitere Möglichkeit sehe ich in einer engeren Zusammenarbeit zwischen den estnisch- und russischsprachigen Schulen Estlands, sei es durch Schüleraustausch oder durch gemeinsame Projekte, Autorenliedfestivals usw. Wie wir aus der Zweisprachigkeitsforschung wissen, müssen für gelingende Zweisprachigkeit Sprachkontakte gefördert und gepflegt werden (Kielhöfer, Jonekeit 1983). Diese Erkenntnis lässt sich auch auf Mehrsprachigkeit übertragen.

Die deutsche Sprache gehört zu den wichtigsten Fremdsprachen nach Englisch und Russisch. Mitte der 1990er Jahre ist in Estland sogar ein kurzzeitiger Anstieg der Zahlen von Deutschlernenden festzustellen. Der Fall des Eisernen Vorhangs und die Wiedervereinigung Deutschlands sorgten für zahlreiche kulturelle Begegnungen und attraktive wirtschaftliche Kontakte. Plötzlich stieg die Nachfrage nach Deutschkenntnissen auf dem Arbeitsmarkt. So erlebt die deutsche Sprache eine Blütezeit im Schuljahr 1997/98, als mehr als ein Fünftel $(22,4 \%)$ aller estnischen SchülerInnen Deutsch lernen. Danach nimmt das Interesse am Deutschlernen kontinuierlich ab. Laut Statistiken haben im Schuljahr 2000/2001 43447 SchülerInnen (20,9\% von allen Lernenden) Deutsch gelernt. Im Jahr 2008/2009 geht die Zahl auf 25095 zurück. Im Schuljahr 2019/20 gibt es nur noch 11628 Deutschlernende (haridussilm.ee).

\section{Diskussion und Ausblick}

In Europa sticht Estland als eines der wenigen Staaten hervor, in denen zwei Fremdsprachen bis zu 9 Jahren gelernt werden (Europäische Kommission 2017: 35). Man könnte auf den ersten Blick behaupten, Mehrsprachigkeit sei in der 
Estnischen Republik erfolgreich implementiert: Das Sprachlernangebot im Curriculum ist vielfältig: Als erste Fremdsprache kann man zwischen Englisch, Deutsch, Französisch oder Russisch wählen. Wenn wir uns jedoch an die eingangs zitierte Definition der curricularen Mehrsprachigkeit besinnen: das Angebot unterschiedlicher Optionen im Curriculum für die Entfaltung individueller Mehrsprachigkeitsprofile, haben wir m.E. noch einen weiten Weg vor uns. Wie Tarvas (2017) mit Recht behauptet, stehen diese vier Sprachen bedauerlicherweise den Lernenden bzw. ihren Eltern nur theoretisch als erste Fremdsprachen zur Auswahl, weil viele Schulen im letzten Jahrzehnt aus ökonomischen Gründen nur Unterricht in Englisch als erste und in Russisch als zweite Fremdsprache eingeführt haben. Obwohl frühes Deutsch an 10 Kindergärten in Tartu (von insgesamt 17 in ganz Estland) angeboten wird, ist das Erlernen des Deutschen als erste Fremdsprache in der Universitätsstadt Tartu nur an einer Privatschule möglich (haridussilm.ee). Wie man aber am Beispiel des Deutschen Gymnasium Kadriorg in Tallinn deutlich sehen kann, sind die Leistungen dieser Schüler und Schülerinnen, die in der Schule Deutsch als erste Fremdsprachen gelernt haben, auch im Englischen hervorragend (Sõstar 2018). Im Schuljahr 2019 haben 33\% ihrer Absolventen im Englischen das Niveau C1 und 6\% sogar das Niveau C2 erreicht (haridussilm.ee). Dies lässt sich als Indiz dafür deuten, dass dadurch bessere Voraussetzungen für das gute Sprachniveau in mehreren Fremdsprachen geschaffen werden, wenn man die schulische Fremdsprachenlernkarriere mit einer anderen Fremdsprache als Englisch beginnt.

Auf lange Sicht ist die Vormachtstellung des Englischen in unseren allgemeinbildenden Schulen in vielerlei Hinsicht nicht förderlich. Sehr gute Deutschkenntnisse sind in einigen Berufsfeldern wie Geschichtswissenschaft, Rechtswissenschaft und Geisteswissenschaften aus historischen Gründen einfach unumgänglich. Ich sehe in meinem A1 Kurs an der Universität Tartu vor allem Studierende der Jura, Philosophie und Geschichte, die Deutsch für ihre Karriere als wichtig ansehen, für die es aber schwierig sein wird, neben dem Hauptfach noch ein hohes Sprachniveau zu erwerben.

Es bleibt nur zu hoffen, dass der aktuelle Entwicklungsplan für den Sprachbereich (Haridus- ja Teadusministeerium 2017: 13) in die Tat umgesetzt wird, dem zufolge die Wahl auch weiterer Fremdsprachen, neben dem Englischen und Russischen, als erste und zweite Fremdsprache gefördert werden sollte.

\section{Merje Miliste}

merje.miliste@ut.ee

University of Tartu

ESTONIA 
MILISTE

\section{Literaturangaben}

Bender, R. 2017. „Babel“ im Baltikum. Die drei Ortssprachen Deutsch, Estnisch und Russisch in deutschbaltischen Lebenserinnerungen. - S. Pasewalck, A. Bers, R. Bender, Hrsg., Zum Beispiel Estland. Das eine Land und die vielen Sprachen. Göttingen: Wallstein, 52-66.

Eesti põhi-ja keskhariduse riiklik õppekava, 1996. - Riigi Teataja, 65-69.

Europäische Kommission/EACEA/Eurydice. 2017. Schlüsselzahlen zum Sprachenlernen an den Schulen in Europa. Luxemburg: Amt für Veröffentlichungen der Europäischen Union.

Feldmann, A. 2013. Fernsehkinder. Einfluss der Medien auf den Fremdsprachenerwerb im Kindesalter. Semesterarbeit. - http://docplayer.org/20114501-Fernsehkinder-einfluss-der-medien-auf-den-fremdsprachenerwerb-im-kindesalter.html (28.10.2020).

Gümnaasiumi riiklik õppekava, 2011. - https://www.riigiteataja.ee/akt/129082014021 (28.10.2020).

Haridus- ja Teadusministeerium, Hrsg. 2009. Eesti võõrkeelte strateegia 2009-2015. Haridus- ja teadusministri käskkiri 13. märts 2009 nr 186.

Haridus- ja Teadusministeerium, Hrsg. 2017. Eesti keelevaldkonna arengukava 2018 2027. Keel loob vä̈rtust. - https://www.hm.ee/sites/default/files/eesti_keelevaldkonna_arengukava_2018-2027_eisi_20.12.2017_0.pdf (28.10.2020).

Haridus- ja Teadusministeerium, Hrsg. 2020. Eesti keele arengukava 2021-2035. https://www.hm.ee/sites/default/files/htm_eesti_keele_arengukava_ 2021-2035_eelnou_02.10.2020_web.pdf (28.10.2020).

haridussilm.ee - https://www.haridussilm.ee/ (28.10.2020).

Innove $2019=2019$. aasta riigieksamite tulemuste kokkuvõte. - https: //www. innove.ee/wp-content/uploads/2019/11/Riigieksamite-ylevaade-2019-2.pdf (28.10.2020).

Neuner, G. 2004. Zur Entwicklung einer Didaktik der curricularen Mehrsprachigkeit. - K.-R. Bausch, F. G. Königs, H.-J. Krumm, Hrsg., Mehrsprachigkeit im Fokus. Arbeitspapiere der 24. Frühjahrskonferenz zur Erforschung des Fremdsprachenunterrichts. Tübingen: Gunter Narr Verlag, 173-180.

Kangor, I. 2002. Saksa keele ainenõukogult. Saksa keelt õpib viiendik õpilastest. Õpetajate Leht, 15.02 .

Kielhöfer, B., Jonekeit, S. 1983. Zweisprachige Kindererziehung. Stauffenburg: Stauffenburg Verlag.

Königs, F. G. 2003. Mehrsprachigkeit ernst genommen. Überlegungen zum Übersetzen (und Dolmetschen) im Fremdsprachenunterricht mit Lernern unterschiedlicher Muttersprachen. - Les langues maternelles dans l'enseignement des langues étrangères/Muttersprachen im Fremdsprachenunterricht/Mother tongues in foreign language teaching. Colloque des 12-13 février, 1999. Lyon: ENS, 83-106.

Krashen, S. D., Terrell, T. D. 1983. The Natural Approach: Language Acquisition in the Classroom. Oxford: Pergamon Press. 
Curriculare Mehrsprachigkeit am Beispiel des Deutschen in Estland

Krumm, H. J. 2003. Sprachenpolitik und Mehrsprachigkeit. - B. Hufeisen, G. Neuner, Hrsg., Mehrsprachigkeitskonzept - Tertiärsprachenlernen - Deutsch nach Englisch. Strasbourg: Council of Europa Publishing, 35-49.

Laar, M. 2017. Streifzug durch die estnische Geschichte. Tallinn: Grenader.

Läänemets, U. 1994. Eesti kooliõpilase keelenõudlus. - Haridus, 4, 49-52.

Mohr, A. 2001. Deutschunterricht und Germanistikstudium in Estland. - G. Helbig (u.a), Hrsg., Deutsch als Fremdsprache. Ein internationales Handbuch. Reihe: Handbücher zur Sprach- und Kommunikationswissenschaft. Bd. 19. Halbband 2. Berlin, New York: Walter de Gruyter, 1683-1690.

Orav, A. T. 2020. Välistööjõud Eestis: mis tööd nad teevad ja kust nad pärit on? https://www.delfi.ee/news/paevauudised/eesti/graafik-valistoojoud-eestis-mistood-nad-teevad-ja-kust-nad-parit-on?id=89966015 (28.10.2020).

Põhikooli ja gümnaasiumi riiklik õppekava, 2002. - https://www.riigiteataja.ee/ akt/174787 (28.10.2020).

Põldvere, M. 2003. Saksa keele õppe ja eesmärgiseade areng Eesti üldhariduskoolides 1919-2002. Tallinn: Avita.

Sõstar, K. 2018. Kadrioru saksa gümnaasium tähistab saksa keel õpetamise 55. aastapäeva. - Oppetajate Leht, 21.09, https://opleht.ee/2018/09/kadrioru-saksa-gumnaasium-tahistab-saksa-keele-opetamise-55-aastapaeva/ (28.10.2020).

Tarvas, M. 2017. Saksa keele olümpiaadist ja saksa keelest laiemat. - Õpetajate Leht, 03.04, https://opleht.ee/2017/04/saksa-keele-olumpiaadist-ja-saksa-keelest-laiemalt/ (28.10.2020).

Võlli, K. 2018. Sadakond lugu keelekümblusest. SA Innove. 